\title{
Correction to: Molecular \& Diagnostic Imaging in Prostate Cancer
}

\author{
Heide Schatten
}

\section{Correction to:}

H. Schatten (ed.), Molecular \& Diagnostic Imaging in Prostate

Cancer, Advances in Experimental Medicine and Biology

1126, https://doi.org/10.1007/978-3-319-99286-0_12

This book was inadvertently published with incorrect volume number 1096. Now, the volume number has been updated as 1126 throughout the whole book.

The updated online version of the book can be found at https://doi.org/10.1007/978-3-319-99286-0 